\begin{tabular}{l} 
DE DE GRUYTER \\
\hline
\end{tabular}

\title{
DYNAMICAL TEMPERATURE FROM THE PHASE SPACE TRAJECTORY
}

\author{
ANDRÁS BARANYAI \\ Department of Theoretical Chemistry, Eötvös Loránd University, P. O. Box 32, Budapest, 1518, \\ HUNGARY
}

In classical statistical mechanics the trajectory in phase space represents the propagation of a classical Hamiltonian system. While trajectories play a key role in chaotic system theory, exploitation of a single trajectory has yet to be considered. This work shows that for ergodic dynamical systems the dynamical temperature can be derived using phase space trajectories.

Keywords: temperature, statistical mechanics

A Hamiltonian system of $N$ mass points is related to the microcanonical ensemble of statistical mechanics when the volume, $V$, and the energy, $E$, of the system are fixed. A microscopic state of the system is a point in phase space represented by a $6 N$-dimensional vector, $\boldsymbol{\Gamma} \equiv\left(\mathbf{q}_{1}, \mathbf{q}_{2}, \ldots \mathbf{q}_{N}, \mathbf{p}_{1}, \mathbf{p}_{2}, \ldots \mathbf{p}_{N}\right)$. The time evolution of this point is described as a trajectory in phase space. In microcanonical ensembles, since the energy is fixed, the evolution of the system is restricted to a $6 N-1$ dimensional hypersurface. The logarithm of the area of this hypersurface yields the entropy, $S$, of the system. Since the entropy of a stable thermodynamic system is a monotonically increasing function of the internal energy, the accessible phase space increases with energy. This provides the opportunity to determine the thermodynamic derivative, $(\partial S / \partial E)_{V, N}=1 / T$, where $T$ is the absolute temperature.

The trajectories within the limit of infinite time perfectly cover the $6 N-1$ dimensional hypersurface according to the ergodic hypothesis. This means that the length of the trajectory for a period of time, $\tau$, is proportional to the microcanonical entropy:

$$
S \propto k \ln \left(\lim _{\tau \rightarrow \infty} \int_{0}^{\tau} d t \sqrt{\dot{\Gamma}^{2}}\right)
$$

Eq.(1) is a line integral measuring the length of the path of the moving system in phase space. This equation can be rewritten as:

$$
S \propto k \ln \left(\lim _{\tau \rightarrow \infty} \int_{0}^{\tau} d t \sqrt{\left(\frac{\partial H}{\partial \boldsymbol{\Gamma}}\right)^{2}}\right)=k \ln \left(\lim _{\tau \rightarrow \infty} \int_{0}^{\tau} d t\left|\frac{\partial H}{\partial \boldsymbol{\Gamma}}\right|\right) .
$$

Let us consider two Hamiltonian systems, $H$ and $H^{\prime}$, with only a minor difference between their energy parameters, $E$ and $E^{\prime}$. The distance covered in phase space by these systems is different because the two energies belong to different areas of the hypersurface to be covered by the trajectories. If the difference in entropies between the two systems is calculated, the quotient in the argument of the logarithm makes it possible to use equality instead of a mere proportionality.

$$
\begin{aligned}
& S\left(E^{\prime}\right)-S(E)=k \ln \left(\frac{\left(\lim _{\tau \rightarrow \infty} \int_{0}^{\tau} d t\left|\frac{\partial H^{\prime}}{\partial \boldsymbol{\Gamma}}\right|\right)}{\left(\lim _{\tau \rightarrow \infty} \int_{0}^{\tau} d t\left|\frac{\partial H}{\partial \boldsymbol{\Gamma}}\right|\right)}\right)= \\
& =k \ln \left(\frac{\frac{\partial H^{\prime}}{\partial \boldsymbol{\Gamma}} \tau^{\prime}}{\frac{\partial H}{\partial \mathbf{T}} \tau^{\prime}}\right)=k \ln \left(\frac{\left.\frac{\partial H^{\prime}}{\partial \boldsymbol{\Gamma}}\right\rangle}{\left.\frac{\partial H}{\partial \mathbf{T}}\right\rangle}\right) .
\end{aligned}
$$

In Eq.(3) the time integral was replaced with the product of time and the time-average of the integrands. The Hamiltonian, $H^{\prime}$, can be approximated as

$$
H^{\prime} \cong H+\frac{\partial H}{\partial \boldsymbol{\Gamma}} d \boldsymbol{\Gamma}
$$

Then

$$
\frac{\partial H^{\prime}}{\partial \boldsymbol{\Gamma}} \cong \frac{\partial H}{\partial \boldsymbol{\Gamma}}+\frac{\partial^{2} H}{\partial \boldsymbol{\Gamma}^{2}} d \boldsymbol{\Gamma}
$$


Using Eq.(5), Eq.(3) can be rewritten as

$$
\begin{aligned}
S\left(E^{\prime}\right)-S(E) & \cong k \ln \left(\frac{\left|\frac{\partial H}{\partial \boldsymbol{\Gamma}}\right|+\frac{\partial^{2} H}{\partial \boldsymbol{\Gamma}^{2}} d \boldsymbol{\Gamma}}{\left|\frac{\partial H}{\partial \boldsymbol{\Gamma}}\right|}\right) \cong \\
\cong k & \left.\frac{\frac{\partial^{2} H}{\partial \boldsymbol{\Gamma}^{2}} d \boldsymbol{\Gamma}}{\left|\frac{\partial H}{\partial \boldsymbol{\Gamma}}\right|}\right) .
\end{aligned}
$$

In the second equality of $E q .(6)$, the $\ln (1+x) \cong x$ approximation was exploited.

To obtain the temperature, division with the energy difference should be included:

$$
\begin{aligned}
& \frac{S\left(E^{\prime}\right)-S(E)}{E^{\prime}-E} \cong k \frac{\left.\frac{\frac{\partial^{2} H}{\partial \boldsymbol{\Gamma}^{2}} d \boldsymbol{\Gamma}}{\left|\frac{\partial H}{\partial \boldsymbol{\Gamma}}\right|}\right)}{\left|\frac{\partial H}{\partial \boldsymbol{\Gamma}} d \boldsymbol{\Gamma}\right|}= \\
&=k\left(\frac{\frac{\partial^{2} H}{\partial \boldsymbol{\Gamma}^{2}}}{\left|\frac{\partial H}{\partial \boldsymbol{\Gamma}}\right|^{2}}\right)=\frac{1}{T} .
\end{aligned}
$$

The dynamical temperature was derived first by Rugh as a time average of $\nabla \cdot\left(\nabla H /\left.\nabla H\right|^{2}\right)$ on the energy surface [1].

$$
\frac{1}{k T}=\left\langle\frac{\nabla \cdot \nabla H}{|\nabla H|^{2}}\right\rangle .
$$

Later, Butler et al. applied a similar approach and derived Eq.(8) only for the configuration part of the phase space and also checked the performance of the method numerically [2]. The essence of both derivations was to transform the phase-space vector, $\boldsymbol{\Gamma}$, from system $E$ into $\Gamma^{\prime}$ of system $E^{\prime}$ by a vector containing the Hamiltonian gradient. In this way arelationship was established between the phase-space points of the two systems.

Our result uses the average speed of evolution of trajectories in phase space, therefore, a Hamiltonian gradient is not required to connect the two sets of phasespace points. Expansion of the energy in Eq.(4) is sufficient to relate the two trajectories. Certainly, the results are identical which proves the validity of this alternative approach while, at the same time, it is a nice example of ergodicity: the method which uses the difference in area of hypersurfaces in an ensembleaverage fashion leading to results that are identical to the method that uses relative trajectory propagation in time.

The explicit form of Eq.(7) can be written as

$$
\frac{1}{k T}=\left\langle\frac{\sum_{i=1}^{N} 3 / m+\nabla_{\mathbf{q}}^{2} \Phi}{\sum_{i=1}^{N} \mathbf{p}_{i}^{2} / m+\left|\nabla_{\mathbf{q}} \Phi\right|^{2}}\right\rangle,
$$

where $\Phi$ is the position-dependent potential in the Hamiltonian and $m$ is the mass of a particle. Eq.(9) contains two terms that feature both in the numerator and denominator. The quotient of the first terms is the well-known kinetic temperature and the quotient of the second terms is the configurational temperature. In actual calculations these two temperatures can be calculated contrary to the full form of Eq.(9) which contains dimensional discrepancies in both the numerator and denominator. The configurational temperature calculation was suggested as an algorithmic check for Monte Carlo computer simulations where the Boltzmann temperature is an input parameter [2].

For completeness, the third, very simple method of deriving the configurational temperature is mentioned [3].The previous derivations did not state whether the two temperatures, the kinetic and configurational, are equal. Using a trivial derivation it is shown that the two quotients are equal [3]. A further advantage of this method is that there is no need for the condition of $N \rightarrow \infty$ to accept the validity of a microcanonical result in terms of the canonical ensemble. The temperature from the momenta of the particles is

$$
T=\frac{1}{3 N k m} \sum_{i=1}^{N} \mathbf{p}_{i}^{2}
$$

If this temperature remains constant over time:

$$
\dot{T}=C \sum_{i=1}^{N} \mathbf{p}_{i} \dot{\mathbf{p}}_{i}=C \sum_{i=1}^{N} \mathbf{p}_{i} \mathbf{F}_{i}=0,
$$

where, for the sake of simplicity, the constant factor is denoted in front of the sum as $C$ and $\mathbf{F}_{i} \equiv-\left(\partial \Phi / \partial \mathbf{q}_{i}\right)$ is the Newtonian force, it is also possible to write that

$$
\begin{aligned}
& \ddot{T}=C \sum_{i=1}^{N}\left(\dot{\mathbf{p}}_{i} \mathbf{F}_{i}+\mathbf{p}_{i} \dot{\mathbf{F}}_{i}\right)= \\
& =C \sum_{i=1}^{N}\left(\sum_{\alpha=x, y, z} F_{\alpha i}^{2}+\frac{\partial \mathbf{F}_{i}}{\partial \alpha} \frac{p_{\alpha i}^{2}}{m}\right)=0 .
\end{aligned}
$$

At equilibrium in isotropic systems the different Cartesian directions are equivalent and there is no correlation between velocities and position-dependent quantities. Thus, 


$$
\frac{3 m}{\left\langle p^{2}\right\rangle} \equiv k T=\frac{\left\langle\nabla_{\mathbf{q}}^{2} \Phi\right\rangle}{\left\langle\left|\nabla_{\mathbf{q}} \Phi\right|^{2}\right\rangle} .
$$

Eq.(13) can be written in this form when $\langle T\rangle$ is manipulated. The results would be the same. Therefore, if the expectation value of the temperature is constant, the kinetic and configurational temperatures are equivalent.

As for the temperature derivation from the phasespace trajectory it is important to note that the $\tau \rightarrow \infty$ condition is essential. Finite segments of trajectories do not cover the hypersurface completely and only contain minimal information about the temperature and force distributions of the system.

\section{REFERENCES}

[1] Rugh, H.H.: Dynamical approach to temperature, Phys. Rev. Lett., 1997 78(5), $772-774$ DOI:10.1103/PhysRevLett.78.772

[2] Butler, B.D.;Ayton, G.A.;Jepps, O.G.;Evans, D.J.: Configurational temperature: Verification of Monte Carlo simulations, J. Chem. Phys., 1998 109, 65196522 DOI:10.1063/1.477301

[3] Baranyai, A.: On the configurational temperature of simple fluids, J. Chem. Phys., 2000 112, 39643966 DOI:10.1063/1.480995 